\title{
Aminosäuren und deren Bedeutung für die Gesundheit
}

\section{Autorin}

Henrike März

\section{Schlüsselwörter}

Aminosäuren, Eiweißverdauung, Nahrungsmittel, L-Arginin, Leber, Neurotransmitter, Immunonutrition, L-Cystein, Haarausfall, L-

Glutamin, Darmbarrierefunktion, L-Lysin, L-Tryptophan, Serotonin

\section{Bibliografie}

OM - Zs. f. Orthomol. Med. 2020; 18: 17-21

DOI 10.1055/a-1251-6013

ISSN 1611-5562

(c) 2020. Thieme. All rights reserved.

Georg Thieme Verlag KG, Rüdigerstraße 14,

70469 Stuttgart, Germany

\section{ZUSAMMENFASSUNC}

Aminosäuren sind hauptsächlich als Eiweißbausteine bekannt, erfüllen jedoch auch wesentliche Aufgaben im Stoffwechsel, da sie als Vorstufen für bedeutende biologische Verbindungen wie Neurotransmitter, Immunfaktoren oder Verdauungsenzyme fungieren. Weil die Stoffwechselwege ineinandergreifen, hängt die Effektivität einzelner Aminosäuren oft von anderen nutritiven Faktoren und einer intakten Enzymfunktion ab. Dies kann bei verschiedenen Erkrankungen von Bedeutung sein. Kenntnisse über Aufgaben, Stoffwechsel und Vorkommen bestimmter Aminosäuren in Nahrungsmitteln ermöglichen eine gezielte Optimierung der Ernährung zur Erhaltung der Gesundheit und unterstützenden Intervention bei Krankheiten.

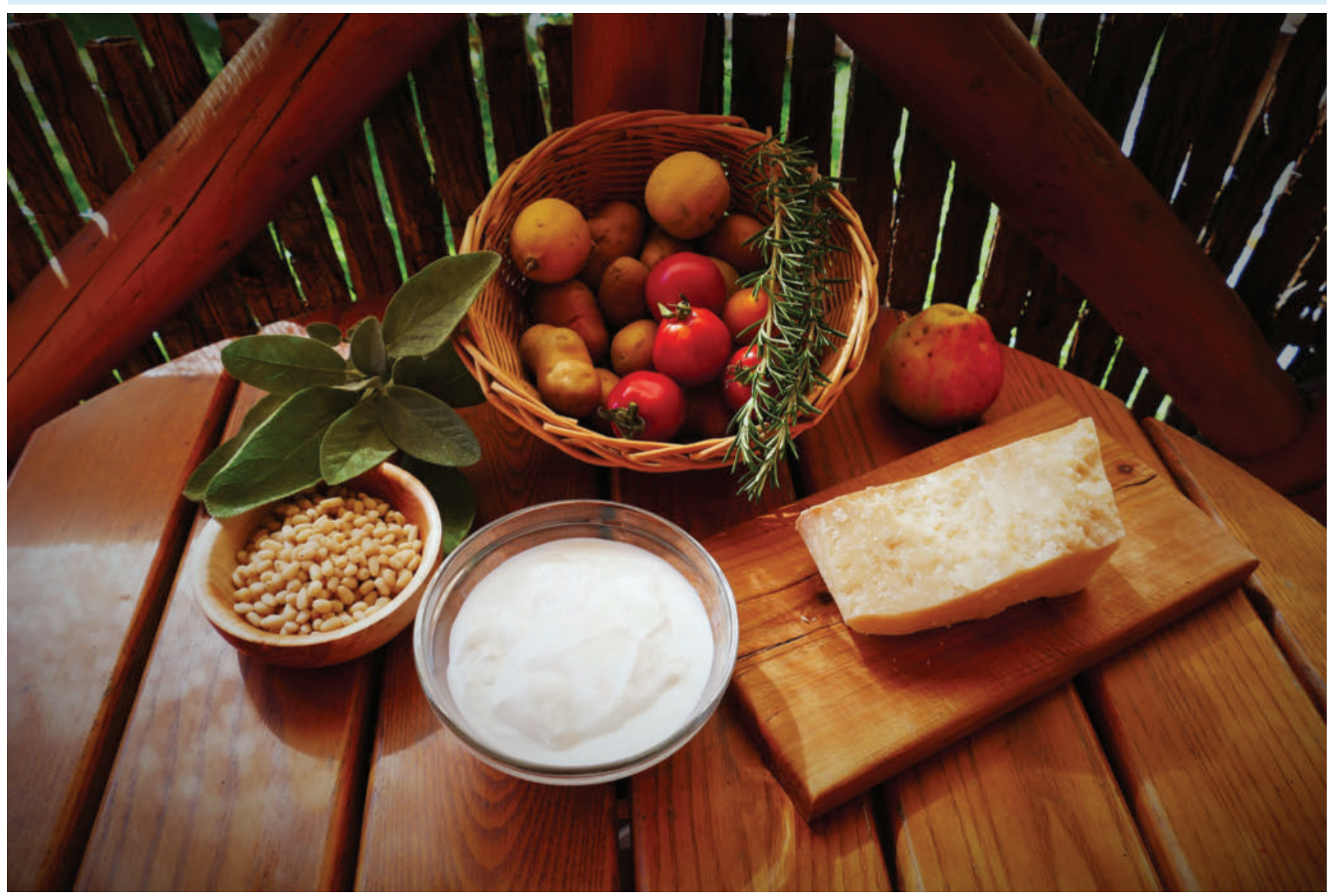

- Abb. 1 In einer gesunden Mischkost findet sich für den gesunden Menschen ein ausgewogenes Maß an Eiweißen. Die mediterrane Ernährung bietet eine gute Orientierung. Quelle: Henrike März 
Unter Aminosäuren wird eine Substanzgruppe stickstoffhaltiger Verbindungen verstanden, die in der Natur weit verbreitet sind. Über 200 Aminosäuren konnten bisher identifiziert werden, wobei manche nur in Pflanzen oder Mikroorganismen vorkommen [1].

Der menschliche Organismus benötigt etwa 20 Aminosäuren für die Bildung körpereigener Eiweiße, weshalb diese Aminosäuren auch als proteinogene Aminosäuren bezeichnet werden. Ihre Anzahl, Reihenfolge und Anordnung im Eiweißmolekül bestimmen über die spezifischen Funktionen des Eiweißes. Manche dieser Aminosäuren werden im Zellstoffwechsel selbst gebildet, andere müssen über die Ernährung zugeführt werden. Heute geht man davon aus, dass 9 der proteinogenen Aminosäuren essenziell sind, d. h. es treten Mangelerscheinungen auf, wenn sie nicht regelmäßig aufgenommen werden. Zu ihnen zählen:

- L-Isoleucin,

- L-Leucin,

- L-Lysin,

- L-Methionin,

- L-Phenylalanin,

- L-Threonin,

- L-Tryptophan,

- L-Valin und

- L-Histidin (nur für Säuglinge).

Zunehmend werden einige Aminosäuren als semi-essenziell bezeichnet. Normalerweise können sie aus anderen Aminosäuren gebildet werden, allerdings kann unter bestimmten Bedingungen, z. B. bei ungenügender Zufuhr der Vorstufen, bei einem Mangel an speziellen Vitaminen, in Stresssituationen (Unfälle, Verletzungen, Infekte), bei Leber- und Nierenerkrankungen sowie bei einer eingeschränkten Enzymfunktion ein Zufuhrbedarf bestehen. Folgende Aminosäuren gelten als semi-essenziell:

- L-Arginin,

- L-Glutamin/L-Glutaminsäure,

- L-Cystein und

- L-Tyrosin.

Weiterhin gehören noch L-Glycin, L-Alanin, L-Serin, LAsparaginsäure, L-Asparagin und L-Prolin zu den proteinogenen Aminosäuren.

Von jeder Aminosäure (Ausnahme: L-Glycin) können aufgrund der Asymmetrie des Moleküls 2 Formen (Isomere) existieren, die entweder der L-Reihe oder der D-Reihe zugeordnet werden. Natürlich vorkommende Aminosäuren gehören bis auf wenige Ausnahmen der L-Reihe an und sollten auch nur in dieser Form eingenommen werden [2].

Da Eiweiße ständig auf- und abgebaut werden, verfügt der Körper über verschiedene Aminosäurepools (Muskulatur, Plasma und Leber), aus denen er Aminosäuren beziehen kann. Die Aminosäuren in den Pools stammen aus der Nahrung, dem körpereigenen Eiweißabbau und der Aminosäureneubildung. Ohne das effektive Aminosäure-Recyclingsystem wäre die hohe Eiweißumsatzrate kaum möglich.

Eine Grundvoraussetzung für die Aminosäureaufnahme stellt eine reibungslose Eiweißverdauung dar. Im Zuge der Verdauungsprozesse werden Eiweiße durch die Einwirkung von Magensäure zunächst denaturiert und anschließend durch bestimmte Enzyme bis hin zu einzelnen Aminosäuren aufgespalten. Durch eine intakte Darmwand können maximal noch 3 miteinander verknüpfte Aminosäuren (Tripeptide) resorbiert werden. Dabei erfolgt der Übergang vom Darminneren ins Blut mithilfe spezifischer Transporteiweiße. Fließen z. B. bei Erkrankungen des Magens oder der Bauchspeicheldrüse die Verdauungssäfte nicht richtig und mangelt es an Enzymen, kann die Eiweißverdauung gestört sein. Dann gelangen größere Eiweißbestandteile in den Dickdarm und werden dort von Darmbakterien zersetzt, wobei Gase und schädliche Abbauprodukte entstehen, die Blähungen, Bauchschmerzen und Durchfall provozieren. Somit hängt die Aminosäureverwertbarkeit von einem gesunden Magen-Darm-Trakt ab.

Mit Ausnahme von Hülsenfrüchten enthält das Eiweiß von pflanzlichen Nahrungsmitteln weniger essenzielle Aminosäuren. Dagegen sind Fisch, Fleisch, Eier und einige Milchprodukte gut mit den notwendigen Aminosäuren bestückt. Optimal erscheint eine Kombination von tierischem und pflanzlichem Eiweiß, wodurch sich die enthaltenen Aminosäuren ergänzen und die biologische Wertigkeit erhöht wird.

Im Folgenden werden ausgewählte Aminosäuren bezüglich ihrer biologischen Funktion und gesundheitlichen Bedeutung näher dargestellt.

\section{L-Arginin}

L-Arginin zählt zu den semi-essenziellen Aminosäuren und entsteht im Harnstoffzyklus, wobei es entweder zur Eiweißbildung herangezogen oder weiter in L-Ornithin umgewandelt wird. Über die Harnstoffbildung entsorgt die Leber schädlichen Ammoniak, sodass L-Arginin indirekt die Entgiftungsfunktion der Leber unterstützt. Steigen die Ammoniakwerte durch eine eingeschränkte Leberfunktion an, schaden sie dem Gehirn. Überhaupt spielt die Leber eine zentrale Rolle im Aminosäurestoffwechsel, was im Fall von Lebererkrankungen berücksichtigt werden sollte. Darüber hinaus kommt L-Arginin im aktiven Zentrum vieler Eiweiße vor und katalysiert aufgrund seiner biochemischen Struktur bes. Phosphorylierungsreaktionen [3].

Aus L-Arginin entsteht Stickstoffmonoxid (NO), das vielfältige Funktionen im Blutgefäß-, Nerven- und 
Immunsystem ausübt, z. B. hängt die Regulation des Blutgefäßtonus von NO ab. Durch die gefäßerweiternde Wirkung kann L-Arginin die Behandlung von peripheren Gefäßerkrankungen, Bluthochdruck und anderer HerzKreislauf-Störungen unterstützen [4]. Außerdem entfaltet NO Neurotransmittereigenschaften und ist im zentralen Nervensystem an der Gedächtnisbildung sowie an der Regulation der Hypothalamus-Hypophyse-Nebennierenrinden-Achse beteiligt [5]. Durch den gasförmigen Zustand benötigt NO keine Rezeptoren, sondern diffundiert direkt durch die Zellwände. Im Gehirn wird mithilfe der neuronalen NO-Synthasen (nNOS), die kalziumabhängig sind, aus L-Arginin NO gebildet. Defekte in diesem Enzymsystem können eine mögliche genetische Ursache für das Restless-Legs-Syndrom (RLS) sein [6]. In einer wissenschaftlichen Studie konnte beobachtet werden, dass verminderte NO- und L-Argininplasmaspiegel mit Schlafstörungen in Verbindung stehen [7]. Bisher liegen jedoch keine Untersuchungen zum therapeutischen Einsatz von L-Arginin bei RLS vor. Immunzellen benötigen ebenfalls NO zur Erregerabwehr, sodass L-Arginin Bestandteil der sogenannten Immunonutrition ist.

Die Bildung des Muskeleiweißes Kreatin hängt ebenfalls von L-Arginin ab, weshalb aktuell auch die Rolle von LArginin bei zehrenden Krebserkrankungen eingehender untersucht wird [8].

Bei der Einnahme von L-Arginin-Präparaten sollte auf die Dosierung geachtet werden, denn ein Zuviel kann zu Blähungen, Durchfällen, Blutdruckabfall und Rötungen im Gesicht (Flush) führen. Außerdem wird eine Kombination mit Antioxidantien (Vitamin C und E) empfohlen, um nitrosativen Stress zu vermeiden [2].

\section{L-Cystein}

L-Cystein gehört neben L-Methionin und L-Cystin zu den schwefelhaltigen Aminosäuren und stellt dadurch eine wichtige Quelle für organisch gebundenen Schwefel dar. Schwefel zählt zu den Mengenelementen, wird jedoch meist nicht extra erwähnt, weil man davon ausgeht, dass seine Zufuhr über die Aufnahme schwefelhaltiger Aminosäuren und Lauchgemüse normalerweise abgedeckt ist. Da Schwefel Schwermetalle bindet, wird L-Cystein z. B. zur Schwermetallausleitung eingesetzt. Gleichzeitig unterstützt es indirekt die Entgiftungsfunktion der Leber, weil es als funktionelle Gruppe von Glutathion an der Inaktivierung oxidativer Verbindungen beteiligt ist [2]. Reduziertes Glutathion stellt neben Vitamin $C$ ein wichtiges Antioxidans im zentralen Nervensystem dar. Dabei verbraucht es für seine eigene Regeneration das B-Vitamin Niacin.

In Kombination mit B-Vitaminen, Zink und L-Lysin findet sich L-Cystein auch in Ergänzungspräparaten gegen Haarausfall. Als Bestandteil des Keratins kommt L-Cystein in Haaren vor. Dabei unterstützt Vitamin $B_{6}$ (Pyridoxalphosphat) den Einbau von L-Cystein in Keratin. Bekannt ist auch die Anwendung von N-Acetylcystein zur Schleimlösung bei Bronchitis.

Im Normalfall kann L-Cystein aus L-Methionin gebildet werden, allerdings setzt das eine gesunde Leberfunktion voraus. Liegt eine Leberzirrhose vor, wird L-Cystein essenziell [9]. Bei der Einnahme sollte eventuell mit Vitamin C kombiniert werden, um die Oxidation zu LCystin zu vermeiden und das damit verbundene Risiko für die Entstehung von Nierensteinen zu minimieren [2]. Darüber hinaus können hohe L-Cystein-Dosen die Blutzuckerregulation bei Diabetikern stören und die Blutgerinnungszeit verkürzen.

\section{L-Glutamin}

Auch wenn L-Glutamin nicht essenziell ist, bildet es den Hauptbestandteil (rund 20\%) des Pools an freien Aminosäuren im Plasma (Normalwert: 500-900 $\mu \mathrm{mol} / \mathrm{l}$ ) [10]. Alle teilungsaktiven Zellen sind auf L-Glutamin als Energiequelle angewiesen, insb. die Darmzellen und Lymphozyten. Dabei hält L-Glutamin die Darmbarrierefunktion aufrecht, weshalb es therapeutisch zur Behandlung des sog. Leaky-Gut-Syndroms („Iöchriger Darm“) eingesetzt wird [11]. Aus Tierversuchen ist bekannt, dass ein L-Glutaminmangel zu Störungen der Darmdurchlässigkeit (intestinale Permeabilität) führt [12]. Des Weiteren konnte beobachtet werden, dass sich bei unterernährten Kindern die Darmbarrierefunktion verbessert, wenn L-Glutamin supplementiert wird [13]. Darmzellen nutzen L-Glutamin als bevorzugtes Substrat, um ihre Funktion und Integrität aufrecht zu erhalten, allerdings sind dabei auch L-Leucin und LArginin von Bedeutung, womit sich wieder das enge Zusammenspiel von Vitalstoffen im menschlichen Stoffwechsel zeigt [14].

Im Gehirn dient L-Glutamin außerdem als Vorstufe für die Bildung des Neurotransmitters $y$-Aminobuttersäure (GABA), die u. a. das Schlafverhalten beeinflusst. Auch ist L-Glutamin eine Vorstufe des endogenen Antioxidans Glutathion und an vielen weiteren Stoffwechselwegen beteiligt. Normalerweise bildet der Körper L-Glutamin mithilfe des Enzyms Glutaminsynthetase im Zytosol der Zellen selbst. Wie bei allen Enzymen können auch hier Defekte vorkommen, die zu einem genetisch bedingten L-Glutaminmangel führen [15].

L-Glutamin findet sich ebenfalls in Immunonutrition-Präparaten, die unterstützend bei Krebspatienten, bei Verbrennungen und nach Operationen eingesetzt werden, um Komplikationen wie Nebenwirkungen der Chemotherapie oder postoperative Infektionen zu reduzieren [10]. Überall da, wo es um zellulären Aufbau, also um Wundheilung geht, wird L-Glutamin vermehrt benötigt. Im 
Normalfall werden Nahrungsergänzungen mit L-Glutamin gut vertragen. Evtl. sollten kleinere Dosen - über den Tag verteilt - einer großen Einzeldosis vorgezogen werden. Bei Menschen, die am Chinarestaurantsyndrom-also einer Glutamatunverträglichkeit leiden - ist Vorsicht geboten.

\section{L-Lysin}

L-Lysin ist eine unentbehrliche Aminosäure, die u. a. im Kollagen vorkommt, also für Haut und Bindegewebe von Bedeutung ist. Sie wird zur Behandlung und Vorbeugung von Haarausfall eingesetzt, weil sie als Bestandteil der inneren Haarwurzel für die Verankerung des Haares in der Kopfhaut sorgt und ihm Struktur gibt [16]. Fehlt LLysin in der Ernährung, kann sich das in dünnen, spröden und schlaffen Haaren und Haarausfall zeigen. Gleichsam spielt in diese Problematik auch der Umstand hinein, dass L-Lysin die Zink- und Eisenresorption fördert [17]. Beide Spurenelemente sind ebenfalls für das Haarwachstum von Bedeutung.

Die Verdauungsenzyme Trypsin und Chymotrypsin können nur bei Vorhandensein von L-Lysin gebildet werden [1]. Sie zählen zu den eiweißspaltenden Enzymen im Dünndarm. Bei L-Lysinmangel leidet demnach die Eiweißverdauung. Auch Wachstumsstörungen und Immunschwäche können auftreten. Die körpereigene L-Carnitinsynthese hängt ebenfalls von L-Lysin ab. L-Carnitin ist wichtig für den Energiestoffwechsel. Der LLysinbedarf wird mit $38 \mathrm{mg} / \mathrm{kg}$ KG angegeben [2]. Insb. Getreideeiweiß ist arm an L-Lysin und auch sonst enthalten pflanzliche Nahrungsmittel eher wenig davon, sodass eine getreidebetonte und/oder vegane Kost zu einem LLysinmangel führen kann.

\section{L-Tryptophan}

Als unentbehrliche Aminosäure ist L-Tryptophan eine Vorstufe des Neurotransmitters Serotonin und des

-Tab. 1 Nahrungsquellen für die Aufnahme ausgewählter Aminosäuren [2].

\begin{tabular}{|c|l|}
\hline Aminosäure & Nahrungsmittel \\
\hline L-Arginin & $\begin{array}{l}\text { Fisch und Meeresfrüchte (z. B. Lachs, Garnelen, Thun- } \\
\text { fisch), Fleisch (z. B. Rind, Schwein), Sojabohnen, Linsen, } \\
\text { Nüsse (z. B. Erdnüsse, Mandeln) }\end{array}$ \\
\hline L-Cystein & $\begin{array}{l}\text { Fisch (z. B. Seezunge), Käse (z. B. Edamer), Fleisch, Soja- } \\
\text { bohnen, Linsen, Steinpilze, Nüsse (z. B. Cashew, Para- } \\
\text { nüsse, Erdnüsse) }\end{array}$ \\
\hline L-Glutamin & $\begin{array}{l}\text { Parmesan, Hühnerfleisch, Haferflocken, Eier, Milch und } \\
\text { Milchprodukte }\end{array}$ \\
\hline L-Lysin & $\begin{array}{l}\text { Käse (z. B. Parmesan), Fisch (z. B. Thunfisch), Fleisch, Soja/ } \\
\text { Tofu, Erdnussmus, Haferflocken }\end{array}$ \\
\hline L-Tryptophan & $\begin{array}{l}\text { Cashewnüsse, Sonnenblumenkerne, Emmentaler Käse, } \\
\text { Kalbsfleisch, Haferflocken, Hühnerfleisch, Thunfisch }\end{array}$ \\
\hline
\end{tabular}

\section{L-Tryptophan}

$$
\downarrow \begin{aligned}
& + \text { Magnesium } \\
& + \text { Niacin } \\
& + \text { Vitamin } B_{6}
\end{aligned}
$$

5-Hydroxytryptophan

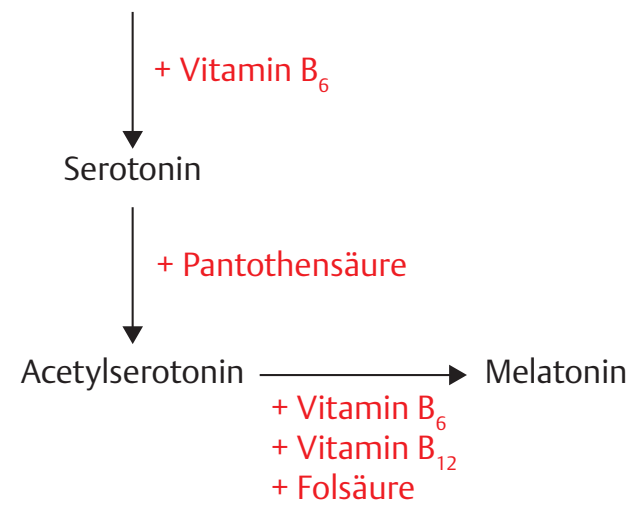

Abb. 2 Serotonin- und Melatoninbildung aus L-Tryptophan und die Beteiligung von Vitalstoffen als Cofaktoren [2].

Hormons Melatonin ( $\mathbf{A} \mathbf{A b} \mathbf{b} \mathbf{2}$ ). Die Verbindungen beeinflussen den Schlaf-Wach-Rhythmus, die Stimmungslage, das Schmerzempfinden und den Appetit. Serotonin wird im Volksmund auch als „Glückshormon" bezeichnet. In wissenschaftlichen Untersuchungen konnte gezeigt werden, dass die Einnahme von 5-Hydroxytryptophan die Serotoninbildung anregt, weshalb es therapeutisch bei Schlafstörungen, Depressionen, aber auch chronischen Schmerzzuständen wie Fibromyalgie und Kopfschmerzen verordnet wird [18].

Der Bedarf an L-Tryptophan wird mit $5 \mathrm{mg} / \mathrm{kg} \mathrm{KG}$ angegeben. Eine normale Mischkost enthält etwa 1-1,5 g L-Tryptophan pro Tag [19]. Interessant ist dabei, dass sich Kohlenhydrate in der Kost durch die notwendige Insulinbildung positiv auf die L-Tryptophanaufnahme ins Gehirn auswirken. Dort konkurriert L-Tryptophan nämlich mit anderen Aminosäuren um den Transport. Die Konkurrenzsituation wird durch Insulin abgeschwächt, indem es die Aufnahme der anderen Aminosäuren in die Muskulatur fördert. Ein weiterer nutritiver Einflussfaktor ist das Koffein. Im Tierversuch konnte beobachtet werden, dass Koffein die Aktivität der L-Tryptophanhydroxylase hemmt [20]. Das Enzym katalysiert die biochemische Umwandlung von L-Tryptophan in 5Hydroxytryptophan. Andauernder Kaffeekonsum könnte deshalb zu einem Absinken der Serotoninwerte im Gehirn führen. Im Gegensatz dazu lässt sich durch körperliche Ausdauerbelastung die Serotoninbildung fördern. 
Von einer selbstverordneten Einnahme ist abzuraten, denn im Neurotransmitterstoffwechsel herrscht ein sensibles Gleichgewicht und komplexes Wechselspiel. Insb. bei Depressionen konnten gegenteilige Wirkungen beobachtet werden, wobei einige Patienten mit Verbesserung, andere allerdings mit einer Verschlechterung reagierten, weil die zugrundeliegenden Ursachen multifaktoriell sind und einer umfassenden Diagnostik bedürfen. Wie bereits dargestellt, lässt sich durch eine veränderte Ernährung und regelmäßige körperliche Bewegung auf natürliche Weise regulierend in den Serotoninstoffwechsel eingreifen, wobei auch an eine optimale Versorgung mit den beteiligten Vitalstoffen, v. a. Vitamin $B_{6}$ und Magnesium gedacht werden sollte.

\section{Fazit}

Der menschliche Organismus ist auf Eiweiße angewiesen. Eiweiße werden auch Proteine genannt, wobei sich der Begriff „Protein“ vom griechischen Wort „proteno“ „ich nehme den 1. Platz ein“ ableitet. In der Tat stellen die in Eiweißen enthaltenen Aminosäuren die einzige vom Menschen verwertbare Stickstoffquelle dar. Wenn es um die Bedarfsdeckung geht, sind v. a. vegane Ernährungsformen kritisch zu betrachten, insb. bei Kindern, die sich im Wachstum befinden. Hierbei muss die Ernährung wohlüberlegt gestaltet und mit Nahrungsmittelkombinationen (z. B. Hülsenfrüchte und Vollkorngetreide) gearbeitet werden, welche die biologische Wertigkeit der enthaltenen Eiweiße erhöhen. In einer gesunden Mischkost, in der die hauptsächlich pflanzliche Nahrung durch natürliche tierische Nahrungsmittel wie Sauermilchprodukte, Fisch, Geflügel, Eier und mageres Fleisch ergänzt wird, findet sich für den gesunden Menschen ein ausgewogenes Maß an Eiweißen. Eine gute Orientierung bei der konkreten Nahrungszusammenstellung bietet die traditionelle mediterrane Kost.

Der ausgleichende Einsatz von Ergänzungspräparaten wird notwendig, wenn Krankheiten (z.B. Leber, Niere, Darm, Allergien) und Funktionsstörungen im Stoffwechsel vorliegen. Da Aminosäuren ihre Wirkung in einem komplexen System mit vielen Einflussfaktoren entfalten, sollten Fachleute zu Rate gezogen werden, die Empfehlungen zur Präparatezusammenstellung und Dosierung geben können.

Autorin

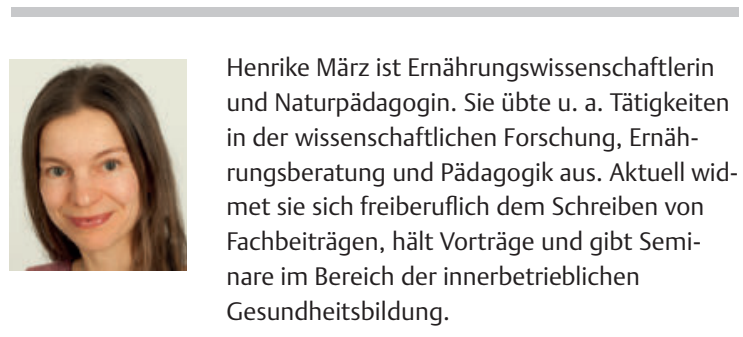

Korrespondenzadresse

\section{Dr. rer. nat. Henrike März}

Im Krautgarten 24

87733 Markt Rettenbach / OT Frechenrieden

E-Mail: henrike-maerz@t-online.de

\section{Literatur}

[1] Buddecke E. Grundriss der Biochemie. 8. Aufl. Berlin: Walter de Gruyter; 1989: 49-53

[2] Zimmermann MB, Schurgast H, Burgerstein UP. Burgerstein Handbuch Nährstoffe. 12. Aufl. Stuttgart: TRIAS; 1982,259275

[3] Fuhrmann J et al. McsB is a protein arginine kinase that phosphorylates and inhibits the heat-shock regulator CtsR. Science 2009; 324: 1323-1327

[4] Boger RH. The pharmacodynamics of L-arginine. J Nutr 2007; 137: $1650-1655$

[5] Kugler HG. Aminosäuren in der Nervenheilkunde. CO'MED 2003; 3

[6] Winkelmann J et al. Variants in the neuronal nitric oxide synthase (nNOS, NOS1) gene are associated with restless legs syndrome. Mov Disord 2008; 23: 350-358

[7] Lavie $L$ et al. Plasma levels of nitric oxide and L-arginine in sleep apnea patients: effects of nCPAP treatment. J Mol Neurosci 2003; 21: 57-63

[8] Albaugh VL et al. Arginine metabolism and cancer. J Surg Oncol 2017; 115: 273-280

[9] Stipanuk MH. Role of the liver in regulation of body cysteine and taurine levels: a brief review. Neurochem Res 2004; 29 : 105-110

[10] Biesalski HK, Fürst P, Kasper H et al., Hrsg. Ernährungsmedizin. 2. Aufl. Stuttgart: Thieme; 1999: 95

[11] Rapin JR et al. Possible links between intestinal permeability and food processing: a potential therapeutic niche for glutamine. Clinics (Sao Paulo) 2010; 65: 635-643

[12] Hulsewe KW et al. Inflammation rather than nutritional depletion determines glutamine concentrations and intestinal permeability. Clin Nutr 2004; 23: 1209-1216

[13] Lima AA et al. Intestinal barrier function and weight gain in malnourished children taking glutamine supplemented enteral formula. J Pediatr Gastroenterol Nutr 2005; 40: 28-35

[14] Marc Rhoads J et al. Glutamine, arginine, and leucine signaling in the intestine. Amino Acids 2009; 37: 111-122

[15] Spodenkiewicz M, Diez-Fernandez C, Rüfenacht V et al. Minireview on glutamine Synthetase deficiency, an ultra-rare inborn error of amino acid biosynthesis. Biology (Basel) 2016; 5: 40

[16] Goluch-Koniuszy ZS. Nutrition of woman with hair loss problem during the period of menopause. Prz Menopauzalny 2016; 15: 56-61

[17] Rushton DH et al. Causes of hair loss and the developments in hair rejuvenation. Int J Cosmet Sci 2002; 24: 17-23

[18] Birdsall TC. 5-Hydroxytryptophan: a clinically-effective serotonin precursor. Altern Med Rev 1998; 3: 271-280

[19] Bosin TR. Serotonin metabolism. In: Essman WB,Ed. Serotonin in Health and Disease: availability, localization and disposition. New York: Spectrum; 1978: 181-300

[20] Lim BV et al. Caffeine inhibits exercise-induced increase in tryptophan-hydroxylases expression in dorsal and median raphe of Sprague-Dawley rats. Neurosci Lett 2001; 308: 25-28 